\title{
The impact of acute kidney injury on midterm outcomes after coronary artery bypass graft surgery: A matched propensity score analysis
}

\author{
Sean Gallagher, MRCP, ${ }^{\mathrm{a}, \mathrm{b}, \mathrm{c}}$ Dan A. Jones, MRCP, ${ }^{\mathrm{a}, \mathrm{b}, \mathrm{c}}$ Matthew J. Lovell, MRCP, PhD, ${ }^{\mathrm{a}, \mathrm{c}}$ Sevda Hassan, MRCP, ${ }^{\mathrm{a}}$ \\ Andrew Wragg, MRCP, PhD, ${ }^{\text {a,c }}$ Akhil Kapur, FRCP, MD, ${ }^{\text {a,b,c }}$ Rakesh Uppal, FRCS, CT/h, ${ }^{\text {a,b,c }}$ and \\ Muhammad M. Yaqoob, MD, FRCP, $\mathrm{PhD}^{\mathrm{a}, \mathrm{b}, \mathrm{c}}$
}

\begin{abstract}
Background: The development of acute kidney injury (AKI) after coronary artery bypass graft (CABG) surgery is associated with increased short- and long-term mortality. Whether AKI has a causal relationship with subsequent mortality or whether the development of AKI simply occurs in patients with more comorbidity undergoing more complex procedures remains unresolved.
\end{abstract}

\begin{abstract}
Methods and Results: This was an observational cohort study of prospectively collected data from 4694 patients discharged from the hospital after first-time CABG surgery at a tertiary cardiac center between 2003 and 2008. AKI was defined using the Risk, Injury, Failure, Loss, and End stage (RIFLE) criteria, which require at least a $50 \%$ increase in serum creatinine. The primary outcome measure was all-cause mortality determined via UK Office of National Statistics. A total of $562(12.0 \%)$ of patients developed AKI after CABG surgery. Patients who developed AKI were older, more likely to be female, and had more comorbidity than patients who did not develop AKI. In a Cox multivariable analysis, the development of AKI was an independent predictor of midterm mortality (hazard ratio, 1.80; 95\% confidence interval, 1.50-2.16). Subsequently, a comparison of 562 patients who sustained AKI with 562 propensity score-matched patients who did not sustain AKI was undertaken. After propensity matching, baseline clinical and operative characteristics were similar between both groups. After Cox multivariable analysis of the propensity-matched cohort, AKI remained an independent predictor of midterm mortality (hazard ratio, 1.52; 95\% confidence interval, 1.19-1.93).
\end{abstract}

Conclusions: The development of AKI after CABG is a serious event associated with worse midterm survival. This excess mortality cannot be explained simply by coexisting comorbidity and surgical complexity. (J Thorac Cardiovasc Surg 2014;147:989-95)

Earn CME credits at

http://cme.ctsnetjournals.org

Acute kidney injury (AKI) is a common problem after cardiac surgery. Depending on definition, AKI may complicate more than $30 \%$ of cardiac operations, ${ }^{1,2}$ with $1 \%$ to $2 \%$ of patients subsequently requiring postoperative dialysis. ${ }^{3-5}$ It is likely that the etiology of AKI after cardiac surgery is multifactorial, with advanced age, preexisting kidney

\footnotetext{
From the Renal and Cardiac Directorate, ${ }^{a}$ Barts and the London National Health Service Trust, William Harvey Research Institute, ${ }^{b}$ Queen Mary and Westfield University, London, and National Institute for Health Research Cardiovascular Biomedical Research Unit, ${ }^{c}$ London Chest Hospital, London, England. Disclosures: Authors have nothing to disclose with regard to commercial support. Received for publication Dec 30, 2012; revisions received Feb 16, 2013; accepted for publication March 6, 2013; available ahead of print April 15, 2013.

Address for reprints: Muhammad M. Yaqoob, MD, FRCP, PhD, Department of Translational Medicine and Therapeutics, William Harvey Research Institute, Barts and The London, Queen Mary's School of Medicine and Dentistry, John Vane Building, Charterhouse Square, London EC1M 6BQ, England (E-mail: m.m.yaqoob@qmul.ac.uk). $0022-5223 / \$ 36.00$

Copyright (c) 2014 by The American Association for Thoracic Surgery http://dx.doi.org/10.1016/j.jtcvs.2013.03.016
}

disease, left ventricular impairment, and procedural complexity with prolonged cardiopulmonary bypass and aortic cross times being important predictors of its subsequent development. ${ }^{3,6,7}$

The need for dialysis after cardiac surgery is a serious event associated with a marked increase in early morbidity and mortality. ${ }^{3}$ Recently, evidence has emerged to suggest that AKI, defined by smaller postoperative increases in serum creatinine $(\mathrm{sCr})$, is also associated with a poor prognosis after cardiac surgery. ${ }^{2,8,9}$ This evidence is primarily derived form retrospective analyses of large cardiac surgical data sets or observational studies and may be confounded by the fact that patients with the highest burden of comorbidity before surgery or those undergoing the most complex operations are most likely to develop postoperative AKI. By virtue of comorbid disease or procedural complexity, these patients are likely to have a worse postsurgical prognosis independent of the development of AKI. Whether AKI has a causal relationship with subsequent mortality or whether the development of AKI simply reflects comorbidity and/or procedural complexity within a high-risk patient population remains unresolved.

We have undertaken an observational single-center cohort study at a tertiary cardiothoracic surgical unit in London, 


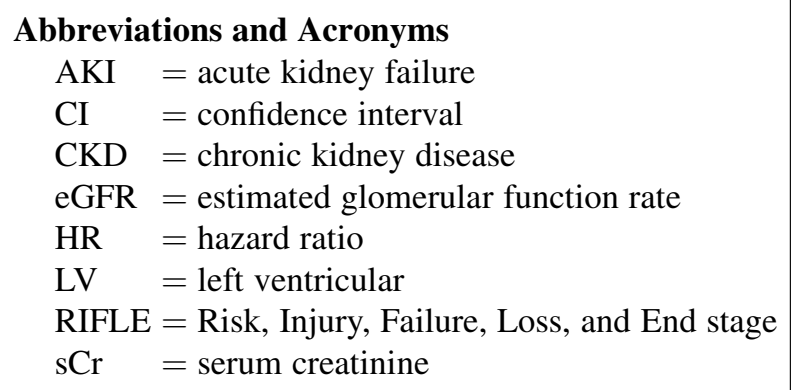

United Kingdom, to evaluate the impact of AKI on survival of patients after coronary artery bypass graft (CABG) surgery. By using propensity score matching, we have minimized the impact of patient comorbidity and surgical complexity on midterm mortality after cardiac surgery.

\section{METHODS \\ Patients and Setting}

This study analyzes consecutive patients undergoing isolated first-time CABG surgery at Barts Health National Health Service Trust, who survived to hospital discharge. During the study period of January 1, 2003, to December 31, 2007, 4891 patients underwent first-time CABG surgery, of whom we excluded 87 with incomplete surgical database records, 35 established on preoperative renal replacement therapy, and 75 who died in the hospital postoperatively. The final study cohort comprised 4694 patients $(96.0 \%$ of all firsttime $\mathrm{CABG}$ operations during the study period) who, for subsequent analysis, were divided into 2 groups: those who developed AKI (AKI group, $\mathrm{n}=562$ ) and those who did not develop AKI (no AKI group, $n=4132$ ) (Figure 1).

We prospectively excluded patients who died in the hospital postoperatively from subsequent analysis. This was to not confound analysis of the effect of AKI on longer-term mortality.

\section{Definition of AKI}

AKI was defined according to the Risk, Injury, Failure, Loss, and End stage (RIFLE) classification by the change in $\mathrm{SCr}$ postoperatively compared with baseline sCr. This classification system requires $\mathrm{sCr}$ levels to increase by at least $50 \%$ from baseline to be classified as AKI. ${ }^{10}$ Baseline $\mathrm{sCr}$ levels were obtained 2 days before $\mathrm{CABG}$ surgery. Postoperatively, $\mathrm{sCr}$ was measured daily for the first postoperative week unless the patient was discharged within this period. Patients sustaining AKI were further stratified according to RIFLE class: RIFLE-R corresponds to a $50 \%$ increase in sCr, RIFLE-I to a $100 \%$ increase in $\mathrm{sCr}$, and RIFLE-F to a $200 \%$ increase in $\mathrm{sCr}$.

\section{Data Collection}

Detailed clinical information was recorded at CABG surgery. This information included age, sex, body mass index, symptom status (New York Heart Association class and Canadian Cardiovascular Society angina status), previous cardiac history (prior myocardial infarction, time since myocardial infarction and prior percutaneous coronary intervention, and preoperative atrial fibrillation), prior medical history (diabetes mellitus, hypertension, peripheral vascular disease, previous stroke or transient ischemic attack, and chronic obstructive pulmonary disease), and baseline renal function $(\mathrm{sCr}$ and estimated glomerular function rate [eGFR]). The GFR was estimated from the Modification of Diet in Renal Disease equation. ${ }^{11}$ Procedural information recorded included the anatomical severity of coronary artery disease, preoperative left ventricular function, and operative data (including use of cardiopulmonary bypass, cross-clamp time, perfusion time, use of internal thoracic arterial grafts, use of preoperative intra-aortic balloon pump, and procedural urgency). Procedural urgency was defined as urgent or elective. Urgent indicated that either medical factors or anatomical severity of coronary artery disease required the patient to stay in the hospital for his or her surgery after coronary angiography. Elective surgery was defined as patients fit to be discharged from the hospital after coronary angiography, with readmission for surgery at a later date.

\section{Outcome Measures}

The primary study end point was all-cause mortality. The UK Office of National Statistics periodically links live/death status of treated patients to our unit's surgical database as part of the National Central Cardiac Audit Database. Study patients were followed up until August 2011 (median follow-up, 5.6 years; interquartile range, 4.2-6.9 years). In addition, early in-hospital outcomes, including perioperative stroke, need for resternotomy, and length of hospital stay, were recorded.

\section{Statistical Analysis and Propensity Score Matching}

All statistical analyses were performed using SPSS, version 18.0 (SPSS, Inc, Chicago, Ill). Baseline patient, procedural, and postoperative characteristics were compared between the 2 groups. Categorical data are summarized using absolute values (percentage). Normally distributed, continuous data are presented as mean $\pm \mathrm{SD}$ or, where skewed, as median (interquartile range). Normally distributed continuous variables were compared using Student $t$ tests, and the Mann-Whitney $U$ test was used to compare nonnormally distributed continuous variables. Categorical data were compared using the Pearson $\chi^{2}$ test.

Baseline comorbidity was unbalanced between the AKI and no AKI groups. A nonparsimonious logistic regression model with AKI as the dependent variable (c-statistic, 0.775 ) was constructed to adjust for the confounding of baseline comorbidity and surgical complexity. Covariates in the model included age, sex, body mass index, previous myocardial infarction, hypertension, previous stroke, peripheral vascular disease, 3-vessel coronary disease, left main stem disease, left ventricular (LV) ejection fraction less than $35 \%$, diabetes mellitus, chronic obstructive airway disease, intra-aortic balloon pump use, internal thoracic artery use, new stroke, baseline sCr, preoperative atrial fibrillation, procedural urgency, and need for resternotomy. To balance comorbidity between the study groups, a greedy matching SPSS macro was used to match the 562 patients who sustained AKI with 562 patients from the "no AKI" group with similar comorbidity. This created a "propensity-matched no AKI" population.

Midterm survival was described using the Kaplan-Meier method, and comparisons were made using the log-rank statistic. Estimations of risk were calculated using Cox regression analysis. Potential independent predictors of outcome were identified by univariate Cox regression analyses, and all significant univariate predictors $(P \leq .05)$ were then entered into the multivariate Cox regression model.

\section{Ethics}

The data were collected as part of a mandatory national cardiac audit, and all patient-identifiable fields were removed before analysis. The local ethics committee advised that formal ethical approval was not required.

\section{RESULTS}

A total of 4694 patients underwent first-time CABG surgery and survived to hospital discharge during the study period. A total of 562 patients (12.0\%) developed an episode of postoperative AKI: of these patients, $342(7.3 \%)$ had RIFLER, $145(3.1 \%)$ had RIFLE-I, and $75(1.6 \%)$ had RIFLE-F.

\section{Patient and Operative Characteristics (Table 1)} Full unmatched study population. Patients who developed AKI were older $(69.4$ vs 66.3 years; $P<.0001)$, more 


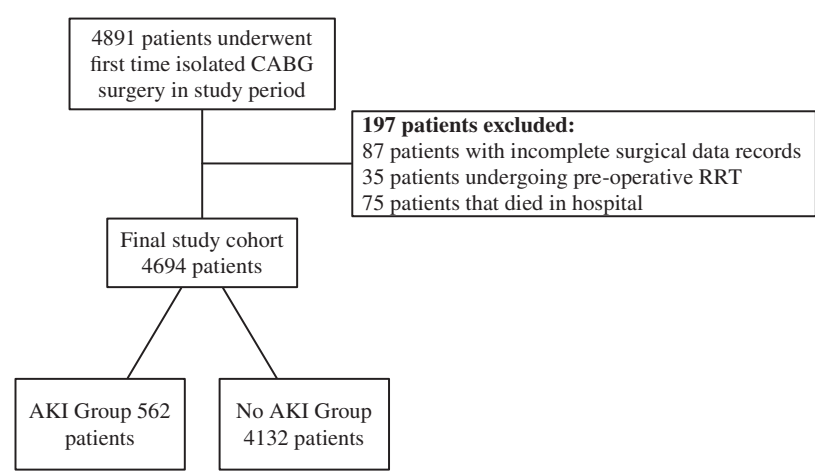

FIGURE 1. Flow diagram of patients undergoing first-time isolated coronary artery bypass grafting $(C A B G)$ between January 2003 and December 2007, describing patient exclusions and incidence of acute kidney injury (AKI). RRT, Renal replacement therapy.

likely to be female $(23.7 \%$ vs $20.0 \% ; P=.041)$, and more likely to have associated comorbidity than patients who did not develop AKI. Notably, patients who developed AKI had more severe preoperative angina symptoms (Canadian Cardiovascular Society class $3-4)(41.5 \%$ vs $34.9 \%$; $P=.002)$ and preoperative heart failure symptoms (New York Heart Association class III-IV) (30.1\% vs $18.8 \%$; $P<.0001)$. Moreover, diabetes mellitus $(46.8 \%$ vs $28.8 \%$; $P<.0001)$, hypertension $(86.8 \%$ vs $77.0 \% ; P<.0001)$, peripheral vascular disease $(20.1 \%$ vs $11.8 \% ; P<.0001)$, previous myocardial infarction $(53.9 \%$ vs $44.3 \% ; P<.0001)$, LV dysfunction (LV ejection fraction, $<50 \%)(48.9 \%$ vs $36.0 \% ; P<.0001)$, preoperative atrial fibrillation $(4.6 \%$ vs $2.4 \% ; P=.003)$, chronic obstructive pulmonary disease $(11.9 \%$ vs $7.9 \% ; P=.001)$, and previous stroke $(9.9 \%$ vs $6.5 \% ; P=.006)$ were significantly more common in the AKI group than the no AKI group.

Patients in the AKI group were also more likely to have undergone urgent CABG surgery $(43.8 \%$ vs $31.0 \%$; $P<.0001)$, to have had a preoperative intra-aortic balloon pump $(2.8 \%$ vs $1.0 \% ; P<.0001)$, and less likely to have received an internal thoracic arterial graft at surgery $(89.1 \%$ vs $91.9 \% ; P=.026$ ) than patients in the no AKI group.

Propensity-matched population. After propensity matching, all baseline patient characteristics were balanced between the 2 groups, except the presence of left main stem disease, which was more common in the propensity-matched no AKI group $(30.6 \%$ vs $24.7 \% ; P=.028)$. Procedural characteristics were also balanced between the 2 groups after matching.

\section{Renal Characteristics (Table 1)}

Full unmatched study population. The mean baseline $\mathrm{SCr}$ and eGFR for all patients were $1.12 \pm 0.34 \mathrm{mg} / \mathrm{dL}$ and $75.0 \pm 26.4 \mathrm{~mL} / \mathrm{min}$, respectively. Patients who developed AKI had significantly higher baseline $\mathrm{sCr}$ (1.24 vs $1.10 \mathrm{mg} / \mathrm{dL} ; P<.0001)$ and lower baseline eGFR (68.9 vs $75.9 \mathrm{~mL} / \mathrm{min} ; P<.0001$ ) than patients who did not develop AKI. Patients with preoperative chronic kidney disease
(CKD) 3 (eGFR, 30-60 mL/min) $(40.6 \%$ vs $27.5 \%)$ and CKD 4 (eGFR, $<30 \mathrm{~mL} / \mathrm{min})(4.3 \%$ vs $0.9 \%)$ were more common in the AKI group than the no AKI group $(P<.0001)$. Peak postoperative $\mathrm{sCr}$ was higher in the AKI group ( 2.58 vs $1.22 \mathrm{mg} / \mathrm{dL} ; P<.0001)$. Only $21(3.7 \%)$ of the patients with AKI required postoperative dialysis.

Propensity-matched population. Baseline $\mathrm{sCr}$ and eGFR were not different, and there was no difference in the proportion of patients with CKD $3(40.6 \%$ vs $38.8 \%)$ or CKD $4(4.3 \%$ vs $2.5 \%)$ between the AKI and no AKI groups after propensity matching. Obviously, peak postoperative $\mathrm{sCr}$ remained different between the groups.

\section{Early Postoperative Outcomes in Full Unmatched \\ Population (Table 1)}

Full unmatched study population. Postoperative stroke $(1.4 \%$ vs $0.3 \% ; P<.0001)$ and need for resternotomy $(5.5 \%$ vs $1.8 \% ; P<.0001)$ were significantly higher in the patients who developed AKI. Furthermore, length of hospital stay was longer for patients who developed AKI when compared with patients who did not develop AKI (14 vs 7 days; $P<.0001$ ).

Propensity-matched population. After propensity matching, there was no difference in postoperative stroke or need for resternotomy between the groups. Length of hospital stay remained longer for patients who developed AKI compared with patients who did not (14 vs 8 days; $P<.0001)$.

\section{Midterm All-Cause Mortality After CABG}

Full unmatched study population. Patients who sustained AKI had higher mortality rates during follow-up (1 year, 8.6\% [95\% confidence interval $\{\mathrm{CI}\}, 3.8 \%$ $15.7 \%$ ]; and 5 year, $24.2 \%$ [ $95 \%$ CI, $18.4 \%-30.4 \%$ ]) than patients who did not sustain AKI (1 year, 2.4\% [95\% CI, $1.2 \%-4.7 \%]$; and 5 year, $10.6 \%$ [95\% CI, $8.5 \%-13.1 \%$ ]) (log rank $P<.0001)$ (Figure $2, A)$.

Mortality rates among patients with RIFLE-R were $6.7 \%$ $(95 \% \mathrm{CI}, 1.7 \%-16.7 \%)$ at 1 year and $22.6 \%(95 \% \mathrm{CI}$, $15.5 \%-30.64 \%$ ) at 5 years. In patients with RIFLE-I, the 1-year mortality rate was $11.8 \%$ (95\% CI, 3.2\%-26.5\%) and the 5-year mortality rate was $28.0 \%(95 \% \mathrm{CI}$, $17.2 \%-38.8 \%$ ), and in patients with RIFLE-F, the 1-year mortality rate was $10.7 \%(95 \% \mathrm{CI}, 1.2 \%-32.7 \%)$ and the 5-year mortality rate was $21.9 \%$ (95\% CI, 8.3\%-39.5\%). Propensity-matched population. After propensity matching, midterm mortality remained higher in the AKI group than the propensity-matched no AKI group (1-year mortality. $5.0 \%$ [ $95 \%$ CI, $1.3 \%-12.6 \%$ ]; and 5-year mortality, $17.3 \%$ [95\% CI, 11.6\%-24.0\%]) (log rank $P=.0008)($ Figure $2, B)$.

\section{Predictors of Midterm All-Cause Mortality After CABG}

Full unmatched study population. The development of AKI (hazard ratio [HR], 1.80; 95\% CI, 1.50-2.16), age 
TABLE 1. Clinical and procedural characteristics of the unmatched and matched study cohorts according to the development of AKI

\begin{tabular}{|c|c|c|c|c|c|}
\hline & No AKI $(n=4132)$ & $\operatorname{AKI}(n=562)$ & $P$ value & $\begin{array}{l}\text { Propensity-matched } \\
\text { no AKI }(n=562)\end{array}$ & $P$ value \\
\hline \multicolumn{6}{|l|}{ Patient characteristics } \\
\hline Age, $y$ & $66.3 \pm 9.6$ & $69.4 \pm 9.2$ & $<.0001$ & $69.3 \pm 8.9$ & .937 \\
\hline Female sex & $825(20.0)$ & $133(23.7)$ & .041 & $149(26.5)$ & .271 \\
\hline BMI & $27.72 \pm 5.31$ & $28.50 \pm 5.85$ & .001 & $28.62 \pm 6.20$ & .740 \\
\hline CCS $3-4$ & $1142(34.9)$ & $233(41.5)$ & .002 & $250(44.5)$ & .306 \\
\hline NYHA III-IV & $778(18.8)$ & $169(30.1)$ & $<.0001$ & $150(26.7)$ & .209 \\
\hline Hypertension & $3182(77.0)$ & $48(86.8)$ & $<.0001$ & $492(87.5)$ & .721 \\
\hline Diabetes mellitus & $1188(28.8)$ & $263(46.8)$ & $<.0001$ & $281(50.0)$ & .281 \\
\hline Previous MI & $1830(44.3)$ & $303(53.9)$ & $<.0001$ & $293(52.1)$ & .550 \\
\hline MI within $30 \mathrm{~d}$ & $467(11.3)$ & $115(20.5)$ & $<.0001$ & $117(20.8)$ & .677 \\
\hline Previous PCI & $419(10.1)$ & $45(8.0)$ & .102 & $61(10.9)$ & .112 \\
\hline COPD & $326(7.9)$ & $67(11.9)$ & .001 & $67(11.9)$ & 1.00 \\
\hline Preoperative atrial fibrillation & $101(2.4)$ & $26(4.6)$ & .003 & $28(5.0)$ & .780 \\
\hline Previous stroke & $267(6.5)$ & $54(9.6)$ & .006 & $58(10.3)$ & .690 \\
\hline Peripheral vascular disease & 489 (11.8) & $113(20.1)$ & $<.0001$ & $111(19.8)$ & .881 \\
\hline LV ejection fraction, $\%$ & & & $<.0001$ & & .411 \\
\hline $35-50$ & $1225(29.6)$ & $213(37.9)$ & & $198(35.2)$ & \\
\hline$<35$ & $264(6.4)$ & $62(11.0)$ & & $55(9.8)$ & \\
\hline 3-Vessel coronary disease & $2781(67.3)$ & $415(73.8)$ & .002 & $417(74.2)$ & .892 \\
\hline Left main stem disease & $1058(25.6)$ & $139(24.7)$ & .656 & $172(30.6)$ & .028 \\
\hline \multicolumn{6}{|l|}{ Procedural characteristics } \\
\hline Procedural urgency & $1281(31.0)$ & $246(43.8)$ & $<.0001$ & $251(44.7)$ & .764 \\
\hline $\mathrm{CPB}$ & $3817(92.4)$ & $526(93.6)$ & .303 & $528(93.6)$ & .805 \\
\hline Perfusion time & $81.1 \pm 40.2$ & $84.0 \pm 39.2$ & .246 & $80.3 \pm 37.1$ & .233 \\
\hline Cross-clamp time & $55.83 \pm 22.5$ & $55.77 \pm 22.1$ & .963 & $56.55 \pm 20.6$ & .602 \\
\hline ITA use & 3798 (91.9) & $501(89.1)$ & .026 & $489(87.0)$ & .269 \\
\hline Preoperative IABP & $40(1.0)$ & $16(2.8)$ & $<.0001$ & $15(2.7)$ & .855 \\
\hline \multicolumn{6}{|l|}{ Renal characteristics } \\
\hline Baseline $\mathrm{sCr}(\mathrm{mg} / \mathrm{dL})$ & $1.11 \pm 0.31$ & $1.24 \pm 0.58$ & $<.0001$ & $1.21 \pm 0.58$ & .388 \\
\hline Baseline eGFR $\left(\mathrm{mL} / \mathrm{min} / 1.73 \mathrm{~m}^{2}\right)$ & $75.9 \pm 26.0$ & $68.6 \pm 28.5$ & $<.0001$ & $68.5 \pm 24.7$ & .931 \\
\hline Baseline CKD class & & & $<.0001$ & & .176 \\
\hline CKD 1 and $2(e G F R>60)$ & 2957 (71.6) & $310(55.1)$ & & $330(58.7)$ & \\
\hline CKD 3 (eGFR 30-60) & $1136(27.5)$ & $228(40.6)$ & & $218(38.8)$ & \\
\hline CKD $4($ eGFR <30) & $39(0.9)$ & $24(4.3)$ & & $14(2.5)$ & \\
\hline Peak sCr & $1.22 \pm 0.41$ & $2.58 \pm 1.24$ & $<.0001$ & $1.38 \pm 0.76$ & $<.0001$ \\
\hline Postoperative dialysis & $0(0)$ & $21(3.7)$ & $<.0001$ & $0(0)$ & $<.0001$ \\
\hline \multicolumn{6}{|l|}{ Early postoperative outcomes } \\
\hline New stroke & $12(0.3)$ & $8(1.4)$ & $<.0001$ & $4(0.7)$ & .246 \\
\hline Return to theater & $75(1.8)$ & $31(5.5)$ & $<.0001$ & $29(5.2)$ & .791 \\
\hline Length of stay & $7(6-11)$ & $14(8-27)$ & $<.0001$ & $8(6-14)$ & $<.0001$ \\
\hline
\end{tabular}

$A K I$, Acute kidney injury; BMI, body mass index; CCS, Canadian Cardiovascular Society; NYHA, New York Heart Association; $M I$, myocardial infarction; $P C I$, percutaneous coronary intervention; $C O P D$, chronic obstructive pulmonary disease; $L V$, left ventricular; $C P B$, cardiopulmonary bypass; ITA, internal thoracic artery; IABP, intra-aortic balloon pump; $s C r$, serum creatinine; $e G F R$, estimated glomerular filtration rate; $C K D$, chronic kidney disease.

(HR, 1.06; 95\% CI, 1.05-1.07), urgent surgery (HR, 1.30; 95\% CI, 1.12-1.52), baseline eGFR (HR, 0.94; 95\% CI, 0.90-0.98), and preoperative comorbidities (diabetes mellitus [HR, 1.38 ; 95\% CI, 1.18-1.61], chronic obstructive pulmonary disease [HR, 1.69; 95\% CI, 1.70-2.08], LV ejection fraction $<35 \%$ [HR, 2.15; 95\% CI, 1.74-2.66], 3-vessel coronary artery disease [HR, 1.23; 95\% CI, 1.03-1.45], and preprocedural atrial fibrillation [HR, $1.52 ; 95 \% \mathrm{CI}$, $1.09-2.12])$ were all associated with significantly increased risk of midterm postoperative all-cause mortality in the unmatched analysis (Table 2).
Propensity-matched population. After propensity score matching, development of AKI remained an independent predictor associated with increased midterm mortality risk (HR, 1.52; 95\% CI, 1.19-1.93) (Table 2).

\section{DISCUSSION}

In this large, single-center cohort of patients undergoing first-time CABG surgery who survived to hospital discharge, the development of AKI was associated with an increase in all-cause mortality during midterm follow-up. By 

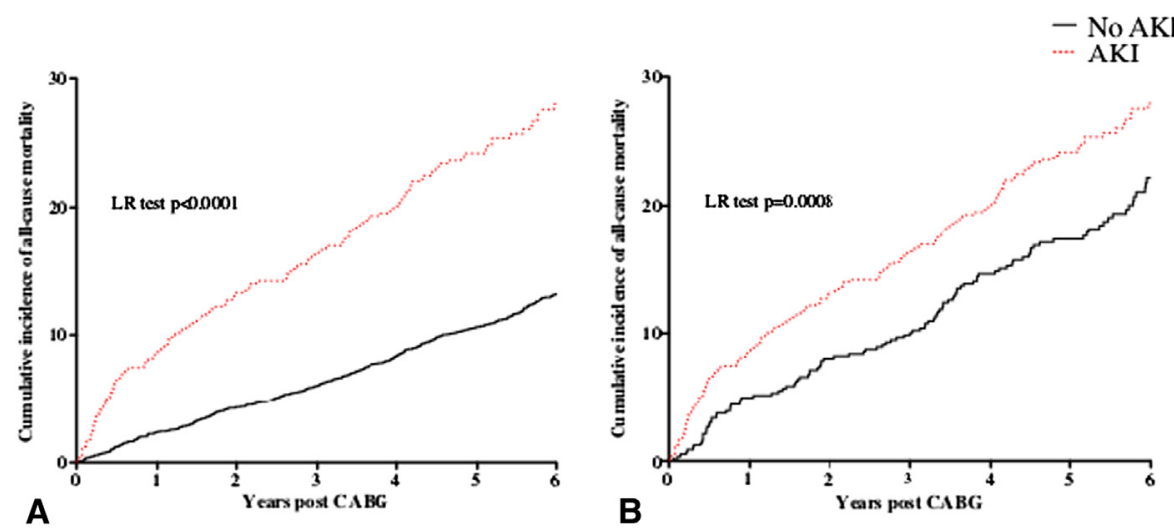

FIGURE 2. Long-term survival of patients with and without acute kidney injury ( $A K I$ ) during hospitalization: unmatched (A) and matched (B) populations. $L R$, Log rank; $C A B G$, coronary artery bypass grafting.

5 years after CABG surgery, an absolute difference in mortality of $13.6 \%$ was evident between patients who sustained AKI and those who did not. Other than poor LV function, developing AKI was the most powerful independent predictor of death in our cohort.

The aim of this study was to investigate the association between perioperative AKI, patient comorbidity and/or surgical complexity, and midterm mortality after cardiac surgery. We were interested in establishing if AKI was an independent predictor of adverse outcome in our cohort. Consistent with our findings, numerous studies have reported that the development of AKI after cardiac surgery

TABLE 2. Independent predictors of long-term mortality in the unmatched and matched study cohorts

\begin{tabular}{lccc}
\hline & $\begin{array}{c}\text { Hazard } \\
\text { ratio }\end{array}$ & $\mathbf{9 5} \%$ CI & $\boldsymbol{P}$ value \\
\hline Unmatched population: Multivariate analysis & & \\
Age (per y) & 1.06 & $1.05-1.07$ & $<.0001$ \\
Development of AKI & 1.80 & $1.50-2.16$ & $<.0001$ \\
Diabetes mellitus & 1.38 & $1.18-1.61$ & $<.0001$ \\
COPD & 1.69 & $1.37-2.08$ & $<.0001$ \\
Preoperative atrial fibrillation & 1.52 & $1.09-2.12$ & .014 \\
LV ejection fraction $<35 \%$ & 2.15 & $1.74-2.66$ & $<.0001$ \\
3-Vessel coronary disease & 1.23 & $1.03-1.45$ & .020 \\
Preoperative eGFR (per 10-mL/min & 0.94 & $0.90-0.98$ & .005 \\
$\quad$ increase) & & & \\
Procedural urgency & 1.30 & $1.12-1.52$ & .001 \\
Matched population: Multivariate analysis & & & \\
Age (per y) & 1.04 & $1.02-1.06$ & $<.0001$ \\
Preoperative atrial fibrillation & 1.63 & $1.05-2.54$ & .030 \\
Previous stroke & 1.62 & $1.15-2.28$ & .006 \\
Procedural urgency & 1.33 & $1.03-1.71$ & .028 \\
LV ejection fraction $<35 \%$ & 2.23 & $1.64-3.04$ & $<.0001$ \\
Development of AKI & 1.52 & $1.19-1.93$ & .001 \\
Baseline sCr & 0.92 & $0.87-0.98$ & .013 \\
\hline AKI Act & &
\end{tabular}

$A K I$, Acute kidney injury; $C O P D$, chronic obstructive pulmonary disease; $L V$, left ventricle; $e G F R$, estimated glomerular filtration rate; $s C r$, serum creatinine; $C I$, confidence interval. is associated with an increase in long-term mortality. $2,4,7,8,12$ Patients with the highest burden of preoperative comorbidity and those undergoing the most complex cardiac surgical procedures more commonly develop postsurgical AKI. ${ }^{3,13-15}$ More important, presurgical and surgical factors may confer a worse postsurgical prognosis, independent of the development of AKI, and, thus, confound previous analyses.

As expected, in our cohort, patients who developed AKI were older, had more comorbid disease and worse preoperative renal function, and were more likely to have undergone urgent surgery. Potentially any or all of these factors may affect subsequent mortality. However, when the effects of comorbidity and surgical complexity were controlled in a multivariable model, the development of AKI still remained a powerful independent predictor of mortality (HR, 1.80). To further assess the association between AKI and postoperative mortality, a propensity score matching algorithm was used to match comorbidity and surgical complexity between patients who developed AKI and those who did not. This matching approach achieved 2 wellbalanced groups for both comorbidities and surgical factors. After matching for comorbidity, the development of AKI still remained associated with a significant increase in 5-year mortality (24.2\% vs $17.3 \%$ ). Because no matching process can eliminate all differences between 2 groups of subjects, multivariable analysis was used to control for remaining differences in baseline comorbidity. After multivariable analyses, AKI remained an independent predictor of midterm mortality risk in the propensity-matched cohort (HR, 1.52).

To our knowledge, this is the first study to use a propensity score matching approach to assess the association between postoperative AKI and midterm mortality. Although propensity score matching is usually used to account for treatment selection bias in observational studies, it can also be used to compile patient cohorts, with and without an exposure, who are equivalent in all other baseline factors. 
Propensity score matching was performed using AKI as the dependent variable, without knowledge of midterm mortality outcomes, just as investigators remain blinded during a randomized clinical trial. The propensity score matching algorithm allowed patient and surgical factors associated with the development of AKI to be balanced between the AKI and the matched no-AKI control group. The mortality in the propensity score-matched no-AKI group was higher than that seen in the unmatched population no-AKI group. This confirms the midterm prognostic importance of the comorbid conditions associated with the development of AKI that we incorporated into the propensity score algorithm. However, despite the use of these statistical techniques, we acknowledge that a cause-and-effect relationship between postoperative AKI and midterm mortality cannot be established with our observational data alone. Of all the factors that we studied, AKI is a powerful predictor of mortality after CABG. Only poor preoperative LV function carried a greater risk of death than developing AKI.

When interpreting our results, the early morbidity rates for our study cohort appear low. This finding is explained by our choice to prospectively exclude patients who died in the hospital postoperatively from our subsequent analysis. A significant proportion of these patients developed AKI. These patients represented a group with multiorgan failure (data not shown), rather than a primary renal pathological condition. Inclusion of these patients with multiorgan failure who died early would have confounded any subsequent analysis of the effect of AKI on midterm mortality.

AKI after cardiac surgery most likely results from a multifactorial renal insult that includes ischemia-reperfusion injury, the systemic inflammatory response to cardiopulmonary bypass, perioperative hemodynamic instability, microembolism, and/or the perioperative administration of nephrotoxins (eg, nonsteroidal anti-inflammatory drugs, angiotensin-converting enzyme inhibitors, or iodinated contrast media). ${ }^{16}$ Why AKI is associated with long-term mortality is incompletely understood. In some patients, the surgical procedure may result in irreversible renal injury and the development of CKD, which is itself independently associated with subsequent mortality. ${ }^{17}$ Postoperative $\mathrm{sCr}$ is a powerful predictor of long-term mortality after cardiac surgery. ${ }^{18}$ However, AKI with complete resolution of renal function (measured by $\mathrm{sCr}$ ) is also associated with an increase in long-term mortality, although several of these patients may subsequently develop CKD. ${ }^{2,19}$

Our study confirms AKI as a common problem after cardiac surgery, and that the development of this complication may identify patients with an increased risk of subsequent mortality after hospital discharge. There are no established prophylaxes or therapies proved to reduce the incidence or the mortality associated with this condition. We hope this study will stimulate the closer postdischarge monitoring of patients who sustain AKI after cardiac surgery. There is little specific follow-up of these patients. AKI may result in ongoing progressive renal damage beyond the acute renal insult, despite the normalization of $\mathrm{sCr}$. This is an important message for cardiologists, cardiac surgeons, and renal physicians responsible for the care of these patients after cardiac surgery. Although future studies are needed to determine the optimal postdischarge follow-up and management of patients who sustain perioperative AKI, we believe that aggressive therapy to optimize control of cardiovascular risk factors should form the mainstay of the current management for these high-risk patients.

\section{Study Strengths and Limitations}

The main strength of this study is that it objectively assesses outcomes after CABG in a large contemporary cohort of consecutive patients. This means that our results are relevant to a broad patient population. Prospectively collected data on short-term morbidity were collected in addition to the primary end point of all-cause mortality.

There are several limitations to this study. First, our primary end point is all-cause mortality, rather than cardiac death, and it is possible that other disease processes could have affected outcome. Second, as with all observational cohort studies, it was open to residual bias and unknown confounding factors. The propensity score-matching algorithm allowed the generation of comparable study groups, but our c-statistic for the algorithm suggests that there may be missing covariates that could affect longer-term mortality after CABG surgery. The purpose of multivariable analysis of the propensity-matched cohort was to correct for any residual differences in baseline comorbidity. Third, we have no data on postoperative urine output. Urine output, in addition to $\mathrm{sCr}$, forms the basis of the RIFLE classification system. It is possible that some patients who did not meet RIFLE sCr criteria would have been reclassified as having sustained AKI if urine output data were available. Fourth, the risk of AKI after cardiac surgery is increased when coronary angiography and surgery occur in close succession. We have no data on timing of coronary angiography in relation to cardiac surgery. Because all elective patients were discharged from the hospital after coronary angiography to wait for admission for cardiac surgery at a later date, it is likely that early cardiac surgery after coronary angiography only occurred in patients undergoing urgent cardiac surgery. Procedural urgency was incorporated within our propensity-matching model and, thus, was evenly balanced between our AKI and propensity-matched no AKI groups. Finally, patients who sustain AKI after cardiac surgery may be less likely to receive beneficial cardiovascular risk modification therapies before hospital discharge (most notably, angiotensin-converting enzyme inhibitors in patients with LV dysfunction). We have no data on patient medical therapy at hospital discharge. If these therapies are not 
subsequently optimized during outpatient follow-up, this may affect long-term outcome.

\section{CONCLUSIONS}

In our large, single-center observational study, the development of AKI after CABG surgery is independently associated with worse midterm survival, both in our unmatched and propensity score-matched cohorts. Our multivariable models and propensity score matching approaches suggest that this excess mortality after hospital discharge cannot be explained simply by preoperative comorbidity or surgical complexity.

We thank colleagues in the cardiothoracic surgical department at Barts Health National Health Service Trust, including Ian Weir, Wael Awad, Kit Wong, Alex Shipolini, Stephen Edmondson, and Kulvinder Lall, and colleagues from the biochemistry department, including Warwick De Ste Croix and Sally Benton.

\section{References}

1. Brown JR, Kramer RS, Coca SG, Parikh CR. Duration of acute kidney injury impacts long-term survival after cardiac surgery. Ann Thorac Surg. 2010;90:1142-8.

2. Hobson CE, Yavas S, Segal MS, Schold JD, Tribble CG, Layon AJ, et al. Acute kidney injury is associated with increased long-term mortality after cardiothoracic surgery. Circulation. 2009;119:2444-53.

3. Chertow GM, Lazarus JM, Christiansen CL, Cook EF, Hammermeister KE, Grover F, et al. Preoperative renal risk stratification. Circulation. 1997;95:878-84.

4. Chertow GM, Levy EM, Hammermeister KE, Grover F, Daley J. Independent association between acute renal failure and mortality following cardiac surgery. Am J Med. 1998; 104:343-8.

5. Chertow GM, Burdick E, Honour M, Bonventre JV, Bates DW. Acute kidney injury, mortality, length of stay, and costs in hospitalized patients. J Am Soc Nephrol. $2005 ; 16: 3365-70$.
6. Rao V, Weisel RD, Buth KJ, Cohen G, Borger MA, Shiono N, et al. Coronary artery bypass grafting in patients with non-dialysis-dependent renal insufficiency. Circulation. 1997;96:II-38-43; discussion II-44-5.

7. Thakar CV, Worley S, Arrigain S, Yared JP, Paganini EP. Improved survival in acute kidney injury after cardiac surgery. Am J Kidney Dis. 2007;50:703-11.

8. Lassnigg A, Schmidlin D, Mouhieddine M, Bachmann LM, Druml W, Bauer P, et al. Minimal changes of serum creatinine predict prognosis in patients after cardiothoracic surgery: a prospective cohort study. J Am Soc Nephrol. 2004;15: 1597-605.

9. Ryckwaert F, Boccara G, Frappier JM, Colson PH. Incidence, risk factors, and prognosis of a moderate increase in plasma creatinine early after cardiac surgery. Crit Care Med. 2002;30:1495-8.

10. Bellomo R, Ronco C, Kellum JA, Mehta RL, Palevsky P. Acute renal failure - definition, outcome measures, animal models, fluid therapy and information technology needs: the Second International Consensus Conference of the Acute Dialysis Quality Initiative (ADQI) Group. Crit Care. 2004;8:R204-12.

11. Levey AS, Bosch JP, Lewis JB, Greene T, Rogers N, Roth D. Modification of Die in Renal Disease Study Group. A more accurate method to estimate glomerular filtration rate from serum creatinine: a new prediction equation. Ann Intern Med. 1999;130:461-70.

12. Bihorac A, Yavas S, Subbiah S, Hobson CE, Schold JD, Gabrielli A, et al. Longterm risk of mortality and acute kidney injury during hospitalization after major surgery. Ann Surg. 2009;249:851-8.

13. Brown JR, Cochran RP, Leavitt BJ, Dacey LJ, Ross CS, MacKenzie TA, et al Multivariable prediction of renal insufficiency developing after cardiac surgery. Circulation. 2007;116:I139-43.

14. Thakar CV, Arrigain S, Worley S, Yared JP, Paganini EP. A clinical score to predict acute renal failure after cardiac surgery. J Am Soc Nephrol. 2005;16:162-8.

15. Wijeysundera DN, Karkouti K, Dupuis JY, Rao V, Chan CT, Granton JT, et al. Derivation and validation of a simplified predictive index for renal replacement therapy after cardiac surgery. JAMA. 2007;297:1801-9.

16. Rosner MH, Okusa MD. Acute kidney injury associated with cardiac surgery. Clin J Am Soc Nephrol. 2006;1:19-32.

17. Hillis GS. Renal function and outcome from coronary artery bypass grafting: impact on mortality after a 2.3-year follow-up. Circulation. 2006;113:1056-62.

18. Brown JR, Cochran RP, MacKenzie TA, Furnary AP, Kunzelman KS, Ross CS et al. Long-term survival after cardiac surgery is predicted by estimated glomerular filtration rate. Ann Thorac Surg. 2008;86:4-11.

19. Mehta RH, Honeycutt E, Patel UD, Lopes RD, Shaw LK, Glower DD, et al. Impact of recovery of renal function on long-term mortality after coronary artery bypass grafting. Am J Cardiol. 2010;106:1728-34. 